\title{
Parallel Solving Method for the Variable Coefficient Nonlinear Equation
}

\author{
Liling Shen", \\ Department of Mathematics, Jining Normal University, \\ Ulanqab, 012000 \\ China
}

Received: June 6, 2021. Revised: December 19, 2021. Accepted: January 9, 2022. Published: January 10, 2022.

\begin{abstract}
In view of the inaccuracy of traditional methods for solving nonlinear equations with variable coefficients in parallel, a new method for solving nonlinear equations with variable coefficients is proposed. Using the generalized symmetry group, the variable coefficient of the equation is taken as a new variable which is the same as the state of the original actual physical field. Some relations between variable coefficient equations and their solutions are found. This paper analyzes the meaning of linear differential equation and nonlinear differential equation, the difference between linear differential equation and nonlinear differential equation and their role in physics, and the necessity of solving nonlinear differential equation. By solving the nonlinear equation with variable coefficients, it can be seen that the general methods to solve the nonlinear equation include scattering inversion, Backlund transform and traveling wave solution. Based on the existing methods for solving nonlinear equations with variable coefficients, the homogeneous balance method is combined with the improved truncated expansion method, truncated expansion method and function reduction method, and the Hopf Cole transform and trial function are combined respectively. The above three methods are used to solve nonlinear equations with variable coefficients. Based on KdV Painleve principle, a parallel method for solving nonlinear equations with variable coefficients is proposed. Finally, it is proved that the method is accurate and effective for the parallel solution of nonlinear equations with variable coefficients.
\end{abstract}

Keywords-Nonlinear equation, parallel solving, variable coefficient.

\section{INTRODUCTION}

$\mathrm{W}$ ITH the development of science and technology, research on non-linear problems has run through many fields such as information science, life science, space science, and environmental science. In nonlinear physics and mechanics, complex nonlinear systems are reduced to nonlinear evolution or evolution equations. The relationship between physical quantities is determined by studying the solutions of these nonlinear equations [1]. Therefore, solving non-linear equations has always been an extremely important problem for scientists in the fields of mechanics, physics and other fields.

In the theory of nonlinear partial differential equations, the solution of equations has always been an important topic widely concerned and studied by physicists and mathematicians. Because of its complexity, it is difficult to solve it by a unified method. Finding effective transformations to transform non-linear differential equations into easy-to-solve equations is the core of most accurate solutions and a major task of the $\mathrm{AC}=\mathrm{BD}$ mode. The key to successfully solving complex equations or constructing complex wave solutions is to algorithmize the solution method and realize the mechanization of the solution process with the help of symbolic computing software systems.

Guided by the idea of mathematical mechanization, the exponential function method is used to construct variable coefficient nonlinear evolution equations and nonlinear differential first order difference equations. The algorithm design and specific examples show that these two algorithms are more direct than the Hirota method, which does not involve the complex process of bilinearizing the equations with Hirota bilinear operators, and the obtained solution is general. Thus, it shows its superiority in the multi-wave solution of mechanized structure. In the practical application of the algorithm, new $\mathrm{N}$-soliton solutions of variable coefficient $\mathrm{KdV}$ Painleve equation and variable coefficient (2+1)-dimensional BK system and (1+1)-dimensional Toda lattice equation were obtained. The periodic variable speed linear propagation diagram, multi soliton pursuit diagram, fusion and fission phenomena and asymptotic behavior of the new solution are simulated graphically. A mechanism adaptive algorithm is proposed in [2]. The corresponding algorithm depends on the error between the actual device output and the reference model output, and also on the variable learning rate. The control strategy is based on two steps; The first is to initialize the 
controller parameters with a reduced number of observations. In the second stage, the parameters of the controller are directly adjusted from the training data by tracking error. Simulation results show that the algorithm is simple to implement and can be extended to multivariable systems. Reference [3] uses piecewise linearization technology and numerical calculation method to identify the maglev system model. On this basis, a robust controller is designed, which can withstand $50 \%$ of the displacement around the operation area, and the simulation is carried out. Then, the switching controller technology is used to extend the technology to full-scale operation. Finally, the performance of the designed controller is evaluated by simulation and experiment. The results show that compared with the traditional PID controller, the performance of the controller is always better than the traditional PID controller. Reference [4] analyzes the stability and optimality of a class of PD controlled nonlinear SISO network systems. The systems considered include network systems with input-output relative quadratic and asymptotically stable zero dynamics. A set of semi global stability conditions are derived, which can be interpreted as adjustment criteria. The optimality condition of PD controller is derived by using inverse optimality based on the passivity of appropriate (control) output.

Based on the work of experts and scholars, some constructive methods and symbolic calculations of exact solutions of nonlinear differential equations in the $\mathrm{AC}=\mathrm{BD}$ mode were studied with specific examples, and some results obtained in the example were visually analyzed. At the same time, related issues were resolved. In the process of algorithm design and implementation, we took full advantage of the "human brain + computer" to make up for the shortcomings caused by limited computer capacity and inability to handle infinite things. In recent decades, many effective methods have been constructed, such as the homogeneous equilibrium method, the exponential function method, and the truncated expansion method. However, most of these methods are used to solve constant coefficient nonlinear evolution equations, and the research history of solving variable coefficient nonlinear evolution equations is still very short. From the practical significance of further research, the coefficients of nonlinear evolution equations change with time and space. The variable coefficient nonlinear evolution equation can better reflect real natural phenomena, so it is necessary to study its basic theory. This article is proposed in this context.

\section{MATERIALS AND METHODS}

The solution of nonlinear evolution equations, especially the precise solutions of these equations, is an ancient and very important research topic in theory and application. Over the years, many mathematicians and physicists have done a lot of valuable work, and the methods for solving them have also been continuously developed and improved. Due to some limitations of the constant coefficient nonlinear evolution equation, the variable coefficient nonlinear evolution equation reveals the physical mechanism of the actual problem, and therefore has a wider application prospect.

Because the definite solution of the nonlinear evolution equation is very complex and the nonlinear equation itself has many characteristics, there are still many important equations with practical application value that cannot be solved accurately. The nonlinear evolution equation that has been solved for the exact solution is also obtained through different methods, and there is no unified method. Whether the obtained solution has physical significance needs further verification. Whether these special solutions can be obtained depends to a large extent on the availability of practical solutions. Therefore, the development of the solution process and method constitutes an organic whole of explicit solution research. The solution method is closely related to the development of soliton theory. Constant-coefficient nonlinear equations can only ideally reflect the laws of actual material movement, in fact, the coefficients of these nonlinear evolution equations change with time and space. Only the variable coefficient nonlinear equation can more accurately, objectively, and truly reflect the complex motion change law of actual matter [5]. Therefore, it is more important to study the solution method of the variable coefficient nonlinear equation, because that is more general. However, it is more difficult to solve the variable coefficient nonlinear partial differential equation than the constant coefficient nonlinear partial differential equation, and it is more complicated to study. In recent years, some progress has been made in solving the variable coefficient nonlinear equation.

The linear differential equation has a remarkable feature that its solution satisfies the superposition principle. If two solutions of the nonlinear differential equation are known, and the linear superposition of these two solutions is not necessarily the solution of the original differential equation [6]. Nonlinearity is also the cause of the mutation, because for the linear differential equation, a small change in one variable is unlikely to cause a sudden change in another variable, while the nonlinear differential equation is different. From the mathematical point of view, in function $x=i(a+b)$, the power of the variable is first, and any function composed of variable with the non-first power is nonlinear. For example, $y=a x^{2}+b x+c, y=\sin x$, and $y=\exp i(a+b)$. From the image point of view, the image of the linear function is a straight line (the origin of linearity), and the image of the non-linear function is a curve. The linear function can be considered as a special case of the non-linear function [7].

Physically, physical quantities are functions of space and time, and the relationship between them is always described by differential equations, such as Newton's second law $p=\frac{b^{*} F(x, y)}{a(i-1)}$. For differential equation, linear differential equation means that the power of all variables and their derivatives of each order in differential equation are first. On the contrary, the variables of nonlinear differential equations and their derivatives of any order are not first. From this point of view, linear differential equations are also special cases of non-linear differential equations. Non-linear differential equations reflect the most common and universal laws in nature [8]. 
When studying nonlinear equations, constant coefficient nonlinear evolution equations are just idealizations and approximations of real-world nonlinear problems. In fact, the coefficients of these nonlinear evolution equations change with time and space. The variable coefficient nonlinear evolution equation reveals the physical mechanism of the actual problem, so it has a wider application prospect. However, it is more difficult to solve the variable coefficient nonlinear partial differential equation than the constant coefficient nonlinear partial differential equation, and it is more complicated to study. Each of these non-linear evolution equations has a broad practical background in the field of physics.

An important issue in the study of nonlinear optics is the movement of solitons, especially solitons in optical fibers. In mathematics, the soliton is understood as the local traveling wave solution of the nonlinear evolution equation. The so-called local refers to the case where the solution of the differential equation approaches zero or determines a constant at infinity in space. In other words, solitons refer to stable solitary waves, which have the following properties:

(1) The energy is relatively concentrated.

(2) The elastic scattering phenomenon with particle-like properties when colliding with each other, that is, its waveform, velocity and amplitude can be maintained after encountering similar solitary waves.

(3) It is almost regular everywhere, that is, it is regular except for individual points.

In physics, soliton is understood as a stable, finite energy, non-dispersive solution of the classical field equation. That is, the energy is concentrated in a small area and does not change the waveform and wave velocity after interaction.

Morphologically, solitary waves are coherent structures (or pseudo-ordered structures) that exist in nature. It is a traveling wave that can both propagate through space at a speed of $\mathrm{v}$ and can be at rest.

Solitons: Solitary waves with elastic collision characteristics are called solitons for short. Solitons are quasiparticles that are excited by a non-linear field, do not disperse energy, and are morphologically stable.

In a field theory system, if there is a classical solution that is bound to a limited area at any time, then such solution is called the classical soliton solution. At the same time, the quark field is the basic field, but it is not the lowest state of energy, and the soliton state is the lowest state of energy. Hadrons observed in nature are actual solitons [9]. Soliton theory is a science that studies the exact solution and integrability of non-linear evolution equations. The emergence and development of the soliton concept cannot be separated from the development of natural science and engineering technology, and it is constantly updating its research objects, content and methods. Because it reflects the basic laws of natural phenomena, it also constantly generates new problems and new methods that need to be solved. Because the mathematical problems faced by the soliton theory research are not only diverse and complex, many related disciplines have benefited a lot. With the advent of computers, even extremely complex problems can be easily solved. In today's world where science and technology are highly developed, the study of soliton theory has a vast world, and it has important practical significance in the fields of military, communications, weather forecasting, engineering and technology. Therefore, the study of soliton theory has theoretical and practical significance. There are currently two definitions of solitons:

\section{Definition 1: Soliton}

1) It is a kind of energy limited local solution in wave problems.

2) It can exist stably in a given region of space.

3) Interaction does not change their characteristics.

4) It can be described as a fixed form of wave.

5) It is local, decaying, or constant at infinity.

6) Other solitons can collide strongly with them, and the wave shape will not change.

Definition 2: Soliton

1) It is a traveling wave propagating in one direction.

2) It is distributed in a small area of space.

3) The wave shape does not change with time.

4) The interaction between them has the same elastic collision as particles.

By studying the nonlinear equations with soliton solutions, we can find that these equations can be expressed as:

Sport + dispersion + nonlinearity $=0$,

Or

Sport + dispersion + nonlinearity + dissipation $=0$.

When studying nonlinear equations, constant coefficient nonlinear evolution equations are just idealizations and approximations of real-world nonlinear problems, in fact, the coefficients of these nonlinear evolution equations change with time and space. The variable coefficient nonlinear evolution equation reveals the physical mechanism of the actual problem, so it has a wider application prospect. However, it is more difficult to solve the variable coefficient nonlinear partial differential equation than the constant coefficient nonlinear partial differential equation, and it is more complicated to study. Each of these non-linear evolution equations has a broad practical background in the field of physics. Since the NLS equation is a non-linear equation involving complex numbers, this brings many difficulties to the solution. Scholars have proposed some methods, such as the direct integration method, the inverse scatter method, the modified Jacobian elliptic function expansion method, the Riccati projection equation mapping method, and so on.

\section{RESULTS}

From the perspective of topological properties, solitons can be divided into topological solitons and non-topological solitons. The necessary condition for the existence of topological solitary waves is that they can be simplified and in the vacuum state (ground state), that is, there are different vacuum states at infinity, or different boundary conditions. When there is a soliton solution, the boundary conditions at infinity are different from when there are no solutions. Non-topological solitons do not need to have the above conditions. Regardless of the existence of soliton solutions, they have the same boundary conditions at infinity [10]. In 
general, the positive and negative (dark) solitons and their sequences of the bell-shaped distribution are non-topological, but the Kink solitons are topological solitons (his module or derivative is bell-shaped, such as the basic dark solitons in fiber). It should be noted that the same equation may support two types of solitary wave solutions with different topological properties. For example, the NLS equation supports bright soliton solutions, small amplitude bright-dark soliton solutions (non-topological) and basic dark soliton solutions (topological). Although there is no uniform solution method for nonlinear equations, some special analytical methods have been summarized. These methods also play a very important role in solving the variable coefficient nonlinear equation. The specific methods are:

The scattering inversion method is an important method to accurately solve the nonlinear evolution equation. Usually, the scattering inversion method obtains the solitary wave solution of the nonlinear evolution equation [11]. It played a very important role in the formation and development of the soliton theory. The scattering inversion method is used to solve the initial value problem of KdV Painleve equation. For the KdV Painleve equation:

$\psi=\frac{b u}{a t}-6 u \frac{a u}{b t}+\frac{b y^{2}}{a x^{3}}-1=0$

After the GGKM transformation, it can be transformed into:

$u=\frac{b x}{a y}+p t(x), t=\frac{\ln a x-1}{y(b+i)}$

The GGKM transform actually turns into a Schrödinger equation that the steady-state wave function satisfies in quantum mechanics:

$$
R=u+\frac{\ln a^{2} \psi-i}{\sum p \sin x \exp y}
$$

The nonlinear Schrödinger (NLS) equation is a widely used nonlinear partial differential equation obtained under the special condition of no loss of optical fiber. In recent years, the application of optical solitons in communication has drawn great attention from the academic and industrial circles, and the NLS equation has the advantages of high code rate, long distance, and large capacity. Therefore, it becomes one of the main equations describing optical solitons in optical fibers. At the same time, the nonlinear Schrödinger equation can also be used to discuss the one-dimensional self-adaptation of monochrome waves, the trap phenomenon of nonlinear optics, Langnui waves in the plasma, thermal pulse propagation in solids, superconductors move in electromagnetic fields and the Bose-Einstein condensation effect of the atoms in the laser. Further introducing transformation and selecting an appropriate trial function, the following equation can be obtained:

$\lambda=t \sum \frac{\sin \alpha y^{2}}{\cos \alpha x^{2}} * \int \mathrm{f} f\left[n+R\left(\mathrm{u}-\frac{\ln a x-1}{(b+i)}\right)\right]$

The ground-state soliton that satisfies the equation can be transmitted arbitrarily long distances without distortion in the optical fiber, so the information transmission of the ground-state soliton in the high-frequency optical communication system has aroused great interest.
Unfortunately, the loss in the actual fiber causes the exponential attenuation of the optical pulse power, so the balance between nonlinearity and dispersion effects is disrupted, which affects the transmission of optical solitons. Usually people make the optical fiber parameters (dispersion and non-linearity) slowly change along the longitudinal direction, or use adiabatic amplification methods to maintain soliton transmission in actual optical communication systems.

In an actual optical soliton communication system, the soliton transmission follows the variable coefficient nonlinear Schrodinge equation as follows [12]. The nonlinear partial differential equation $E\left(\mathrm{n}, n_{a}, n_{b}, n_{x}, n_{y}, n_{i}, n_{j}\right)=1$ with two variables was given. The traveling wave solution of the equation was obtained by the $(\mathrm{G} / \mathrm{G})$ expansion method. The specific steps are as follows:

The first step: The partial differential equation is transformed into an ordinary differential equation by a traveling wave transformation, and a traveling wave solution can be obtained by solving the obtained ordinary differential equation. This solution is concise and straightforward, and the analytical solutions obtained are mainly solitary wave solutions and periodic solutions, which reflects the important properties of nonlinear waves. Assume that the traveling wave transformation of the equation is as follows:

$\mathrm{z}=\frac{\left\|E \mathrm{n}-E n_{a}-E n_{b}-E n_{x}-E n_{y}-E n_{i}-E n_{j}\right\|}{v n f f \int \lambda R \cos (x) \sin (y)}$

where $p$ is the undetermined constant. From the characteristics of the transformation law and the variable coefficient equation, it can be seen that in the process of solving the variable coefficient nonlinear equation, the transformation form, law, and solution method have been further studied [13]. Three methods are proposed: the combination of homogeneous balance method and improved truncated expansion method, the combination of truncated expansion method and function integration method, the combination of Hopf cole transformation and trial function, and a variety of new transformation forms are given.

$\Delta k=(z-\lambda) E \mathrm{n} / \mathrm{vn}^{*} \tan (\mathrm{x}, \mathrm{y})$

The second step is to construct the series solution form of nonlinear variable coefficient equation by elliptic function, and then use the balance of nonlinear term and highest derivative term to determine the series term, so as to obtain the solution of nonlinear variable coefficient equation.

A special function is used to construct the series solution of the variable coefficient nonlinear equation. The term of the series is also determined by the balance of the nonlinear term and the highest derivative term, and the variable coefficient nonlinear equation is transformed into a series of equations that can be solved, so as to obtain the solution of the coefficient nonlinear equation.

Suppose $\Delta \varphi$ can be expressed as $f(n)$ polynomials, $\mathrm{a}_{\mathrm{i}}(\mathrm{i}=0,1,2,3, \ldots, n)$ is undetermined constant, $\mathrm{n}$ is determined by the homogeneous balance principle (considering nonlinear characteristic $\mathrm{s}$, dispersion $\mathrm{c}$ and order factor $\mathrm{h}$ of dissipation, 
according to the principle that the highest order term can be partially balanced), which satisfies the following second-order linear ordinary differential equation:

$$
A=\log (\Delta k+\Delta \varphi) / a_{i} \mathrm{I} \frac{f(\mathrm{n})-z}{\|s+c+h\|^{i+1}}
$$

When $\mathrm{i}>0$, the trigonometric solution of the equation is obtained:

$$
G=A \cos \sqrt{\Delta k-1}+a_{i} \sin (\sqrt{\Delta \varphi-i})
$$

A relation between some nonlinear Schrodinger equations with variable coefficients can be established by the generalized symmetric operation and the corresponding finite transformation. If the original equation is a constant coefficient equation, the solution of some types of variable coefficient equation can be obtained by the solution of the constant coefficient equation [14]. The variable coefficient equation is obtained by the finite transformation, and the relation between nonlinear evolution equation and Schrodinger equation can be established by GGKM transformation. The potential energy, i.e. the solution of nonlinear equation, can be obtained by scattering inversion method, which is also the soliton solution. Based on the above algorithm, several groups of common solution curves are further shown for subsequent calculation

Using Jacobi elliptic sine function expansion method, cosine function expansion method, the third type Jacobi elliptic function expansion method and Jacobi elliptic CS expansion method, the envelope periodic solutions are obtained respectively. When the modulus $\mathrm{m}=1$, the corresponding envelope solitary wave solution can be obtained. In case 5, the modified Jacobi elliptic function expansion method is used to obtain two envelope periodic solutions and a simple solution of the equation. Among them, solution one is consistent with Jacobi elliptic sine function expansion method, solution two is consistent with Jacobi elliptic cosine function expansion method, and solution three is a new simple solution, which proves that the modified Jacobi elliptic function expansion method is effective. The specific curve is as shown in Figs. 1-4.

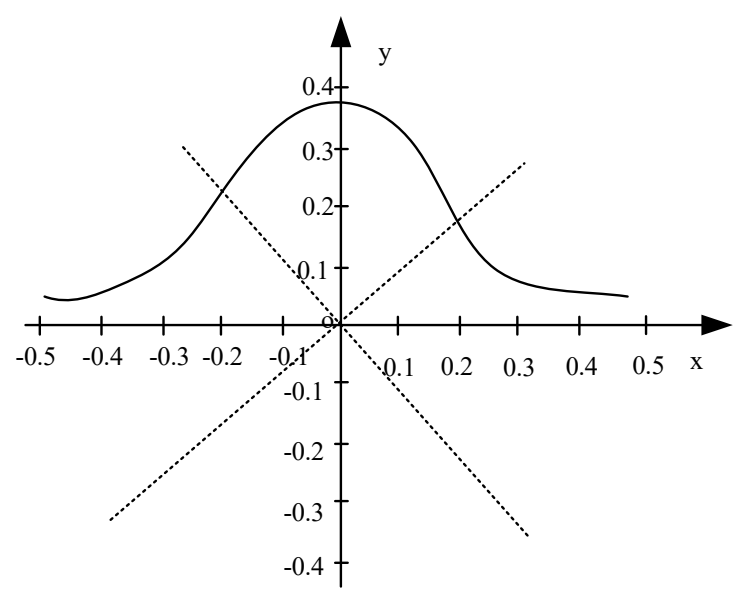

Fig. 1 The curve of bell type (wave packet type) equation

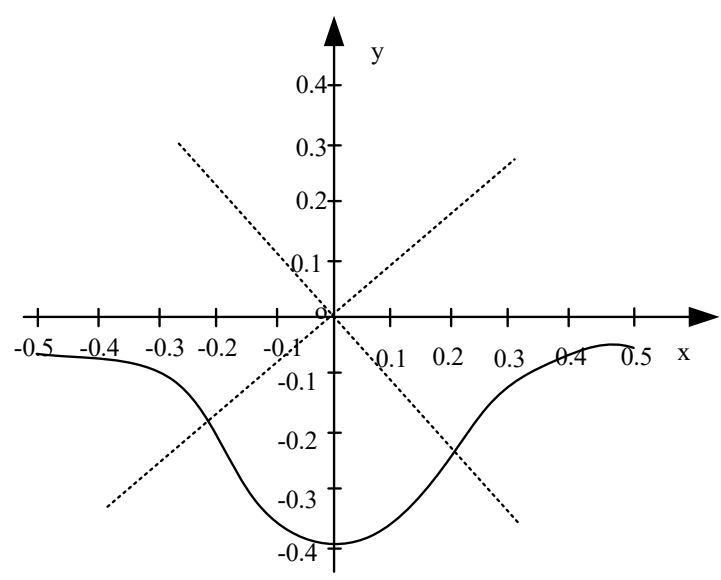

Fig. 2 The curve of anti-bell type (concave type or vortex type) equation

Furthermore, the solution of the equation is indirectly equated as a trigonometric function, the method of solving the exact solution of the equation is called indirect fitting method. Hyperbolic function, elliptic function or other explicit functions are usually used to solve this method, which includes three methods: using constraint equation, using transformation and using operator. This paper focuses on the kink curve of constraint equation, as follows.

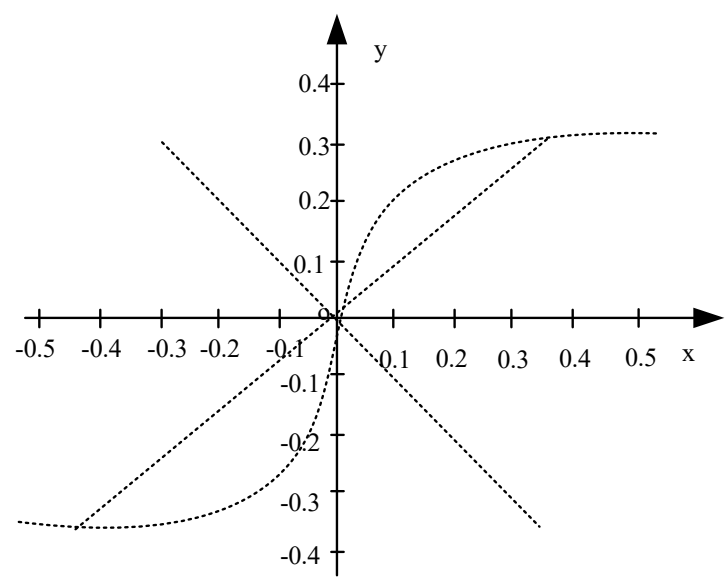

Fig. 3 The curve of kink equation 


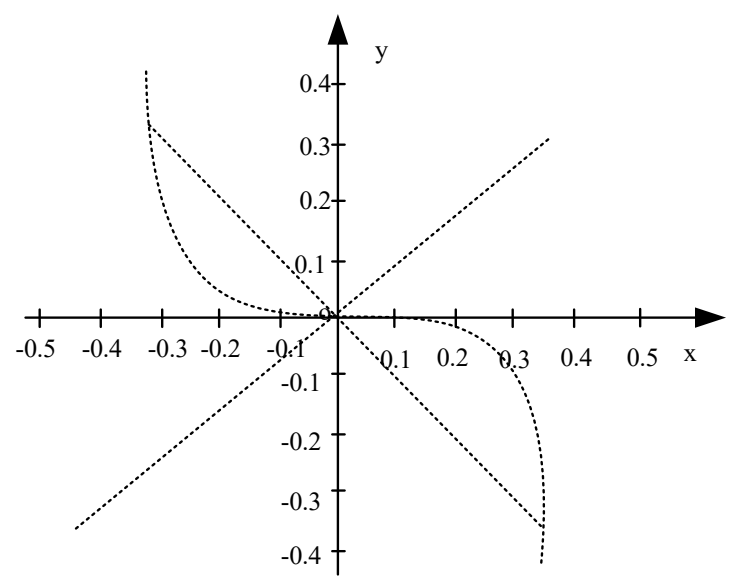

Fig. 4 The curve of anti-kink equation

According to Fig. 1, the curve decreases monotonically when the $\mathrm{x}$-axis is 0.5 and the $\mathrm{y}$-axis is 0.4 , and increases monotonically when the $x$-axis is -0.5 and the $y$-axis is 0.4 , with the maximum value of 0.37 ; In Fig. 2 , when the $x$-axis is 0.5 and the $y$-axis is -0.4 , the curve increases monotonically, and when the $\mathrm{x}$-axis is -0.5 and the $\mathrm{y}$-axis is -0.4 , the curve decreases monotonically, with a minimum value of -0.37 ; In Fig. 3, it increases gradually in the first quadrant and decreases gradually in the third quadrant; In Fig. 4, it increases gradually in the second quadrant and decreases gradually in the third quadrant.

Based on the above principle, the curve of soliton equation is further optimized. The curve of the equation is solved by directly assuming the solution of the equation as trigonometric function, hyperbolic function, elliptic function or other explicit function. The mechanism of soliton is due to the existence of nonlinear term, so the soliton equation is usually related to the spectrum problem. The soliton equation is usually derived on the basis of the spectrum problem with the help of zero curvature equation or lax equation, as shown in Figs. 5-6:

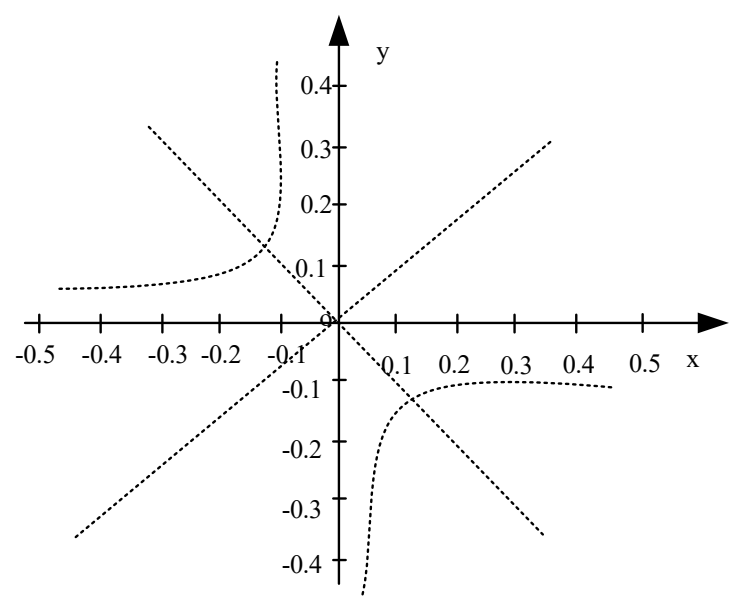

Fig. 5 The curve of singular soliton equation

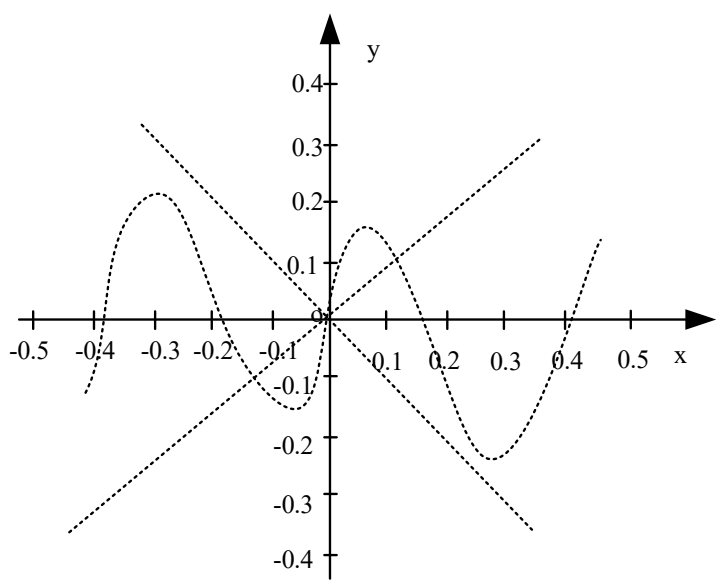

Fig. 6 The curve of (multiple) compact soliton equation

According to Fig. 5 and Fig. 6, starting from the known solution of the soliton equation, a new soliton solution can be obtained, and the new solution can be used as the known solution to obtain an updated solution. A series of solutions of the equation can be generated by repeating the cycle. People gradually discovered that there are some method structures. The BW cklund transform of nonlinear partial differential equations, and the form of the obtained BW cklund transform is completely different. It is very difficult to find a solution for the nonlinear equation, and it is even more difficult to find more solutions. Backlund transformation establishes the relationship between the solution of a nonlinear partial differential equation and the solution of the known linear partial differential equation, or the relationship between two different solutions of a nonlinear partial differential equation [15]. In this way, we can find the solution of the nonlinear partial differential equation according to the known solution of the linear partial differential equation, or find other solutions according to one solution of the nonlinear partial differential equation.

\section{DISCUSSION}

Aiming at the inaccuracy of the traditional parallel solution method of variable coefficient nonlinear equations, a new parallel solution method of variable coefficient nonlinear equations is proposed. In order to judge whether a nonlinear equation is integrable, the method of finding relaxation pairs is usually used. However, there is no unified method to solve various nonlinear equations, so it is difficult to find relaxation pairs. It is proved that $\mathrm{KdV}$ Painleve analysis is one of the most effective methods to judge the integrability of nonlinear equations.

Firstly, the KdV Painleve properties of ordinary differential equations are studied. The integrability of partial differential equations is tested by the differential method of ordinary differential equations (ARS method). If any ordinary differential equation can be simplified by a fully integrable partial differential equation, it must be a $\mathrm{KdV}$ Painleve equation (requiring at most one transformation). The $\mathrm{P}$ and $\mathrm{KdV}$ Painleve experiments (WTC method) of PDE are 
proposed. Due to the unique advantages of KdV Painleve analysis, KdV Painleve property has become a method to define the integrability of nonlinear partial differential equations, that is, KdV Painleve integrability. The parallel solution and transformation of variable precision nonlinear equations are realized. The partial differential equation is transformed into ordinary differential equation by traveling wave transformation, and the traveling wave solution can be obtained by solving the ordinary differential equation. The analytical solutions are mainly solitary wave solutions and periodic solutions, which reflect the important properties of nonlinear waves.

Three new exact solutions of the generalized variable coefficient KdV Painleve equation are obtained by using the expansion method. These solutions are more general and include the analytical solutions given in the literature. Moreover, when A, B and C take different values, there will be many new exact solutions. It can be seen from the above process that this method is suitable for solving a large class of Nonlinear Evolution Equations with variable coefficients. On this basis, the parallel solutions of nonlinear equations with different variable coefficients are verified. The trigonometric function solutions and hyperbolic function curves of different parallel solutions are obtained. Record and draw the detection results of parallel solution curves obtained by different methods.

\section{CONCLUSIONS}

At present, there are many ways to construct the exact solution of nonlinear evolution equation, and the content is complex. On the surface, it seems to have achieved some success in seeking accurate solutions, but to deal with nonlinear terms concisely and clearly, it is far from enough to rely on the existing mathematical tools, which requires us to further explore and find.

The solution of nonlinear problems is an important work in nonlinear science. It is well known that there are infinite solutions to nonlinear partial differential equations, which brings great difficulty to the solution of nonlinear equations. However, most of the nonlinear equations that can better describe the actual physical problems are variable coefficient equations, so it is more difficult to get the exact solution of the variable coefficient nonlinear equation. In many methods of solving nonlinear partial differential equation, most of them are only for constant coefficient nonlinear partial differential equation, but for variable coefficient nonlinear partial differential equation, there are few effective methods at present.

The KdV Painleve equation with non-uniform term, the generalized diffusion model in population problem and the exact solution of $2+1$ dimensional long-short wave equations are studied by the f-expansion. These solutions have different forms: hyperbolic function, trigonometric function and rational function. Because of the arbitrary choice of parameters, more travelling wave solutions of nonlinear PDEs can be obtained by this method. From the point of view of the solution process, the f-expansion method is not only applicable to the constant coefficient nonlinear partial differential equation, but also to the variable coefficient nonlinear equation, and even can be extended to the other variable coefficient nonlinear equation. It provides a powerful method for solving higher order equations. At the same time, the nonlinear Derrida-Lebowitz-Speer-Spohn equation is transformed into a third-order dispersion equation by a nonlinear function transformation, and then the exact solutions with variable form and other forms are obtained by the method of separating variables. All the exact solutions obtained in this paper further enrich the physical application of these four nonlinear partial differential equations.

Aiming at the inaccuracy of parallel solution of nonlinear equations with variable coefficients by traditional methods, a new method for solving nonlinear equations with variable coefficients is proposed in this paper. The compatibility conditions of differential operators and eigenfunctions with arbitrary function forms are introduced, and a new family of KdV Painleve equations with time-dependent coefficients and their L2 pairs are derived. The constant coefficient isospectral KdV Painleve equation family is a special case, which contains many well-known equations, equation families and new equations. Its more general case is a family of variable coefficient non isospectral equations with self-consistent sources. The new exact solution expression of the variable coefficient, the equation family and the non-reflection potential $\mathrm{N}$-wave solution equation are obtained by the backscatter transform. It solves the problem of non isospectral $\mathrm{KD}$, Schrodinger spectrum of equation family, and the problem that scattering data can't solve for a period of time in the research field, so as to resolve the existing contradictions in the existing literature. Through the expression of the solution, the evolution characteristics of the velocity, amplitude and wave width of the solitary wave solution of the variable coefficient KdV Painleve equation family in the process of straight-line propagation, as well as the relationship between the isospectral and non isospectral attributes of the equation itself are established by means of graphic simulation. The reflection coefficient obtained on the basis of the non isospectral KdV Painleve equation family paves the way for solving the non isospectral KDZ equation family, which further improves the scattering theory of the anti-scattering method for solving the non isospectral KdV Painleve equation family.

There is no universal solution to the nonlinear partial differential equation, and especially there is no systematic and effective solution to the variable coefficient nonlinear partial differential equation, which needs to be studied more. The results of these methods are usually traveling wave solutions, and the study of non-traveling wave solutions of nonlinear partial differential equations needs to be further deepened. In addition, some equations are substituted into the equations after wave reduction and homogeneous equilibrium, and the resulting equations have no solution. These cases are also worth studying for a large number of non-linear partial differential equations that have not been solved. These problems are helpful to further understand and improve the solution methods and techniques of nonlinear partial differential equations. It needs constant thinking and exploration, and hopes to achieve more and better results. 
variable-coefficient generalized shallow water wave

\section{ACKNOWLEDGMENT}

The research is supported by: Scientific research project of Higher Education Institution in Inner Mongolia Autonomous Region in 2021 "Mathematics education curriculum group construction and textbooks development in local normal universities under the background of normal major certification, (No. NJSY 21242)".

\section{References}

[1] F. A. Hendi, and M. M. Al-Qarni, "The variational Adomian decomposition method for solving nonlinear two-dimensional Volterra-Fredholm integro-differential equation," Journal of King Saud University-Science, vol. 31, no. 1, pp. 110-113, 2019.

[2] F. X. Xu, S. S. Wang, and F. R. Liu, "Research on RBF neural network model reference adaptive control system based on nonlinear U-model," Automatika, vol. 61, no. 1, pp. 46-57, 2020.

[3] J. X. Wang, L. J. Chen, and L. Yu, "Model predictive control for magnetic levitation ball system based on equivalent input disturbance sliding mode observer," Control Theory \& Applications, vol. 38, no. 1, pp. 137-146, 2021.

[4] P. Xu, and Y. M. Li, "Adaptive fuzzy finite time fault tolerant control for a class of SISO non strict feedback nonlinear systems," Journal of Liaoning University of Technology (Natural Science Edition), vol. 39, no. 3, pp. 141-148, 2019.

[5] N. M. Yazid, K. G. Tay, C. Y. Yee, and A. M. Sudin, "Solving dissipative nonlinear schrodinger equation with variable coefficient using homotopy perturbation method, Proceedings of the International Conference on Computing," Mathematics and Statistics (iCMS 2015), vol. 12, no. 19, pp. 4814-4819, 2017.

[6] G. B. Tang, Y. Yao, L. Wang, S. P. Cui, and Y. Cao, "Relation of damage variable and gas permeability coefficient of concrete under stress," Journal of Wuhan University of Technology (Materials Science Edition), vol. 33, no. 6, pp. 1481-1485, 2018.

[7] H. T. Ryu, B. J. Yi, and Y. H. Kwon, "Analytical model of variable characteristic of coefficient of restitution and its application to ball trajectory planning," Intelligent Service Robotics, vol. 10, no. 1, pp. 13-29, 2017.

[8] S. T. M. Din, T. Zubair, M. Usman, M. Hamid, M. Rafiq, and S. Mohsin, "Investigation of heat and mass transfer under the influence of variable diffusion coefficient and thermal conductivity," Indian Journal of Physics, vol. 92, no. 9, pp. 1-9, 2018.

[9] P. Zhang, and $\mathrm{H}$. Pu, "The error analysis of crank-nicolson-type difference scheme for fractional subdiffusion equation with spatially variable coefficient," Boundary Value Problems, vol. 15, no. 1, pp. 1-19, 2017.

[10] Q. M. Huang, Y. T. Gao, S. L. Jia, Y. L. Wang, and G. F. Deng, "Bilinear bäcklund transformation, soliton and periodic wave solutions for a dimensional equation," Nonlinear Dynamics, vol. 87, no. 4, pp. 2529-2540, 2017.

[11] V. A. Gordin, and E. A. Tsymbalov, "A fourth-order accurate difference scheme for a differential equation with variable coefficients," Mathematical Models \& Computer Simulations, vol. 10, no. 1, pp. 79-88, 2018.

[12]A. Wang, L. Kuang, and X. Zhang, "A study on flow coefficient of combined throttling groove in spool valves," Hsi-An Chiao Tung Ta Hsueh/Journal of Xi'an Jiaotong University, vol. 52, no. 2, pp. 110-117, 2018.

[13]Z. S. Wang, Y. N. Luo, A. H. Li, Y. X. Wei, H. Q. Guo, and F. Yang, "The effect analysis of correlation between variables on the partial coefficient," Journal of Railway Engineering Society, vol. 34, no. 2, pp. 37-42, 2017.

[14] S. Krzyska, and I. Kubiaczyk, "On bounded pseudo and weak solutions of a nonlinear differential equation in banach spaces," Demonstratio Mathematica, vol. 32, no. 2, pp. 323-330, 2017.

[15]M. Daniel, and O. Horst, "A convergent lagrangian discretization for a nonlinear fourth order equation," Foundations of Computational Mathematics, vol. 17, no. 1, pp. 73-126, 2017.

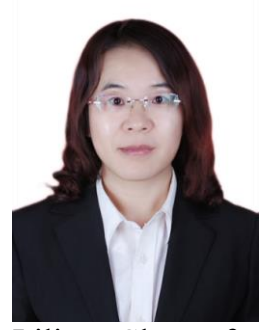

Liling Shen, female, was born in November 1975. Her professional title is associate professor. Her bachelor's degree in mathematics education from Inner Mongolia Normal University in 2001 and her master's degree in mathematics curriculum and teaching from Shandong Normal University in 2006. She is now working in Jining Normal University. Her research fields include mathematics curriculum and teaching and mathematics teacher education. She has published more than 20 academic papers. At the same time, she also presided two Inner Mongolia Autonomous Region-level scientific research projects and four university-level teaching reform projects.

\section{Author Contributions:}

Liling Shen proposed a parallel method for solving nonlinear equations with variable coefficients based on $\mathrm{KdV}$ Painleve principle. Finally, it is proved that the method is accurate and effective for the parallel solution of nonlinear equations with variable coefficients.

\section{Creative Commons Attribution License 4.0 (Attribution 4.0 International, CC BY 4.0)}

This article is published under the terms of the Creative Commons Attribution License 4.0

https://creativecommons.org/licenses/by/4.0/deed.en_US 